\title{
Ueber den Finfluss der relativen Helligkeit und der Farbe des Papieres auf die Sehschärfe.
}

Von

\section{Bruno 盂olbe.}

Hierzu Tafel VIII u. IX.

Die verschiedenen zur Bestimmung der Sehschärfe dienenden Tafeln zeigen einen merklichen Unterschied in der Helligkeit des benutzten Papieres, sowie - wenn auch in weit geringerem Grade — in der Helligkeit oder vielmebr "Schwärze“ der betreffenden Schriftzeichen. Da die von Angenärzten benutzten Tafeln meist frei an einer Wand des Zimmers hängen, so wird durch das Nachdunkeln des Papieres die relative Helligkeit zwischen den Schriftzeichen und dem Grunde bedeutend verändert, wodurch die Sehschärfemessungen, welche mit solchen Tafeln angestellt werden, jedenfalls an Zuverlässigkeit verlieren müssen.

Im Nachstehenden theile ich nun eine Reihe von Sehschärfemessungen mit, bei welchen die photometrisch bestimmte Helligkeit des Grundes $(G)$ und der Lesezeichen $(Z)$ variirt wurde. Da es sich im Laufe der Untersuchung herausstellte, dass die Intensität der Beleuchtung einen grossen Einfluss auf das Verhältniss zwischen der Sehschärfe und der Helligkeitsdifferenz $(G-Z)$ latte, so wurden Kontrolbestimmungen bei verschiedener Beleuchtung angestellt.

\section{Helligkeitsbestimmung von Pigmentflächen.}

Ehe ich an eine Wiedergabe der Beobachtungsresultate gehe, möchte einiges über Helligkeitsmessungen von Pigmentflächen vorausschicken, da meine Untersuchangen darauf basiren.

Eine grössere Reihe von Messungen nach verschiedenen Methoden hatte mir gezeigt, dass zwei im Prinzip ganz verschiedene Methoden mit einer für die Praxis genigenden Genauigkeit die Bestimmung der relativen Helligkeit von Pigmentfarben gestatten. Da ich meine Versuche kürzlich eingehend beschrieben 
Ueber den Einfluss d. relativen Helligkeit u. d. Farbe d. Papieres etc. 563

habe $^{1}$ ), so kann ich mich auf eine kurze Wiedergabe des Wichtigsten beschränken.

Um vergleichbare Resultate zu erhalten, muss man (für die geg. Beleuchtung) ein Helligkeitsmaximum von genügender Konstanz festsetzen. Vergeblich versuchte ich es, durch Bestreichen (mit schwefelsaurem Baryt, Zinkweiss, Bleiweiss etc.) reinweisse Papierflächen von völlig gleicher Oberfächenbeschaffenheit zu erzielen, und nahm daher reinweissen Bristolkarton zum Maassstabe. Da fand ich eine werthvolle Notiz bei König ${ }^{2}$ ), nach welcher eine (weisse!) Papierfläche, welche einige Sekunden über brennendem Magnesiumdraht gehalten worden, sich zum Normalweiss für physiologisch-optische Untersuchungen eigne, indem eine solche Fläche sich stets in ,gleicher Beschaffenheit" herstellen lasse. Da mir nun bezügliche Versuche zeigten ${ }^{3}$ ), dass dieses thatsächlich der Fall ist, so habe ich das König'sche Normalweiss adoptirt und die Helligkeit desselben $\left(h_{N}\right)=100$ gesetzt. Da dieses Normalweiss aber nicht zu direkten Helligkeitsvergleichungen geeignet ist, indem die Oxydschicht sehr locker aufliegt, so benutze ich reinweissen Bristolkarton, dessen Helligkeit ( $\left.W=90,4 \% h_{N}\right)$ durch wiederholte Messungen bestimmt wurde, und reduzire die beobachteten Helligkeitswerthe von Mischfarben (an rotirenden Scheiben) auf Normalweiss. Die in Prozenten der Helligkeit des Normalweiss ausgedrückte Helligkeit eines Pigmentes wollen wir seine Normalhelligkeit nennen. Hat also ein Pigment die Normalhelligkeit $h=n$, so beisst das, dass $h=$ einer Mischung von $n \%$ Normalweiss $+(100-n) \%$ absolut. Schwarz!

Unter allen schwarzen Pigmenten scheint reinschwarzer Seidensammet die geringste Helligkeit zu haben. Nach A ubert's Methode $^{4}$ ) erhielt ich für solchen Sammet $\widetilde{S}=0,25 \% h_{B}$ (Bristol), also $=0,225 \% h_{N}(1 / 440$ des Normalweiss). Aubert (ob. cit. pag. 227 u. f.) hatte für seinen Sammet $h=1 / 500$ des reinweissen

1) Zur Analyse der Pigmentfarben, v. Graefe's Arch. f. Ophth. XXX (1884), 2, pag. 1-68.

2) König: v. Graefe's Arch. f. Ophth. XXX (1884), 2, pag. 162, und Wiedemann's Ann. d. Phys. u. Chem. 1884, Nr. 8, pag. 572.

3) Nachtrag zur Analyse der Pigmentfarben. Daselbst Bd. XXX (1884), 4, pag. $313-314$.

4) A ubert: Die Helligkeit des Schwarz und Weiss. Dieses Arch. f. d. ges. Physiol. XXXI (1883), pag. 224. 
Kartons erhalten. Bei Mischfarben an rotirenden Scheiben diurfen wir die Helligkeit guter schwarzer Sammetsektoren ohne Weiteres $=0$ setzen, da die Helligkeitsdifferenz zwischen $p \% W+$ $(100-p) \%$ schwarzer Sammet und der Normalhelligkeit $(p \%$ $W+[100-p] \%$ absolut. Schwarz) selbst im Maximum nur $0,225 \% h_{N}$ betragen kann, also weit unterhalb der zu erreichenden Genauigkeitsgrenze der Beobachtung liegt. Leider werden die Sammetscheiben bei häufigerem Gebrauche leicht verdriickt, wodurch sehr störende glänzende Stellen entstehen; daher benutze ich lieber den tiefsehwarzen Bristolkarton $\left(S=2,6 \% h_{N}\right)$ und bestimme die Normalhelligkeit der Mischfarbe durch Rechnung oder, was weit bequemer ist und sich mit genügender Genauigkeit ansführen lässt, durch Konstruktion.

Es bedeute $y$ den Prozentsatz Weiss, von der Normalhelligkeit $=W$,

also: $100-y$ den Prozentsatz d. and. Komponente von der Normalhelligkeit $=h$,

[resp. $y^{\prime}(=-y)$ den Prozentsatz Schwarz von der Normalhelligkeit $=S]$,

$\left.x^{1}\right)$ den Prozentsatz Normalweiss von der Normalhelligkeit $h_{N}=100$,

$(100-x)$ den Prozentsatz absolut. Schwarz von der Normalhelligkeit $=0$.

Als Helligkeitsgleichung (an rotir. Scheiben) erhalten wir:

$y \% W+(100-y) \% h=x \% h_{N}+(100-x) \%$ absolut. Schwarz $=100 \%($ Grau $=x)$

(d. h. wir substituiren ein Normalgrau von geforderter Normalhelligkeit).

$$
\begin{aligned}
& y=\frac{100(x-h)}{W-h}=-\left(\frac{100 . h}{W-h}\right)+\left(\frac{100}{W-h}\right) \cdot x . \\
& x=h+\left(\frac{W-h}{100}\right) \cdot y \text {. . . . . . . . . . }
\end{aligned}
$$

Dieses ist, wie man sieht, die Gleichung einer geraden Linie.

Nehmen wir $y$ zur Ordinate und $x$ zur Abscisse, so erhalten wir die gewünschte Reduktionskurve (s. Taf. VIII Fig. 1).

1) Das $x$ entspricht also der Normalhelligheit des resultirenden Grau der Mischung $x \% h_{N}+(100-x) \%$ absol. Schwarz $(H=0)$. 
Ueber den Einfluss d. relativen Helligkeit u. d. Farbe d. Papieres etc. 565

Für $y=0$ ist $x=h$, d. h. gleich der Helligkeit der zweiten Komponente.

Für $y=100$ ist $x=W$, d. h. gleich der Helligkeit des benutzten Weiss.

Letzterer Werth ist von $h$ unabhängig, mithin schneiden sich alle Reduktionskurven, welche (bei verschiedenem $h$ ) einer Mischung mit demselben Weiss entsprechen, in demselben Punkte ( $W$ in Fig. 1).

Bei der Anwendung von Schwarz (S) [für solche Helligkeitsgrade, wo $x<h$ ] erhalten wir:

$$
y^{\prime} \% S+\left(100-y^{\prime}\right) \% h_{b}=100 x
$$

hieraus: $y^{\prime}=-y=\frac{(100 h-x)}{h-S} ; x=h+\frac{h-S}{100} \cdot y . \quad$. (2a a. b) für $\quad y=-100$ ist $x=S$ und für $y=0$ ist $x=h$ ).

Haben wir (für eine gewisse Beleuchtung) die Normalhelligkeit einiger Pigmentflächen bestimmt ${ }^{1}$ ), so können wir leicht durch Rechnung die Normalhelligkeit beliebiger Mischungen der geg. Komponenten (am Farbenkreisel) finden (Formel $1 b$ und $2 b$ ) oder den zu einer verlangten bestimmten Normalhelligkeit $(x)$ nöthigen Prozentsatz Weiss $(+y)$ oder Schwarz $(-y)$ bestimmen (Formel 1a und 2a).

Um alle nöthigen Reduktionen sofort und obne Rechnung auszuftibren, haben wir nur nöthig, die Reduktionskurven auf Millimeter-Coordinatenpapier zu konstruiren, indem wir $y$ (den beobachteten resp. einzustellenden Prozentsatz $W$ oder $S$ ) als 0 rdinate und $x$ (die entsprechende Normalhelligkeit $=$ dem Prozentsatz Normalweiss in der Mischung mit absolutem Schwarz) als Abscisse auftragen. Die Abscisse $x=h$, für $y=0$, giebt die Stelle an, wo die Kurve die Abscissenachse schneidet und entspricht der Normalhelligkeit der einen Komponente. Die Ordinate $y$ hat die Grenzwerthe 0 und $\pm 100 \%$ (resp. $\pm 360^{\circ}$, wenn wir die Sektoren nach Graden messen). Für $y=100 \%$ (resp. $360^{\circ}$ ) ist $x=W$;

1) Bei jeder Stellung einer Pigmentfläche zum auffallenden Lichte giebt es eine gewisse Lage der Blicklinie, für welche die Helligkeit der Pigmentfläche, ceteris paribas, ein Minimum ist. Da die Helligkeit der Fläche mit der Stellung des Beobachters sich bedeutend ändern kann, so ist die Helligkeit von Pigmentflächen stets in der Minimumlage zu messen. [Dieses gilt auch für Messungen am Fettfleck-Photometer]. D. Verf. 
für $y=-100 \%$ (resp. $\left.-360^{\circ}\right)$ ist $x=S$. Der Schnittpunkt der Reduktionskurven für alle Mischfarben mit re inweissem Bristolkarton $(h=90,4)$ liegt in $W$ (Taf. VIII), mit weis sestem Visitenkartenpapier $(h=96,3)$ in $\mathfrak{B}$, mit schw. Bristol in $S$, mit schw. Sammet in $\mathfrak{S}$.

Ganz ebenso können wir verfahren, wenn wir die Mischfarben von geg. farbigen Papieren auf Normalgrau reduziren wollen, z. B. Karmin und Gelb (beides Heidelb. Blmp.). $y=0$, $x=h$ (Karmin) $=17,6$ giebt den Anfangspunkt und $y=100, x=h$ Gelb $=56,9$ den Endpunkt $(G)$ der Reduktionskurve an.

Tab. A. Normalhelligkeit einiger Papiere

(bei diffus. Tageslicht).

Normalweiss (Magnesiumoxyd) $=100$.

[Schw. Sammet . . . . . . . $\mathfrak{S}=0,225$ ],

Sehw. (dicker) Bristolkarton . . . $S=2,6$,

Druckerschwärze . . . . . . . $=3,7$,

Bull's Neutralgrau . . . . . . $=14,3$,

* Neutralgrau (B.-K.), Nr. 541 . . . $N=29,2$,

* Helles Silbergrau, Nr. 462 . . . $=52,4$,

Papier der Snellen'schen Tafeln . $=83,0$,

Reinweisser Bristolkarton . . . . $W=90,4$,

* Papier, Nr. 50 (doppelt genommen) $=92,4$,

Weissestes Visit.-Kart.-Pap. (Bristol) $\mathfrak{B}=96,3$,

Karmin . . . . . . . . . . $K=17,6$,

Scharlach . . . . . . . . . $=24,7$,

Gelb . . . . . . . . . . $G=56,9$,

Gelb (pikrins. Natron) . . . . . $G^{\prime}=46,3$,

Grïn II . . . . . . . . . $G r_{\mathrm{II}}=29,3$,

Ultramarin II . . . . . . . . . $U_{\mathrm{II}}=26,0$,

Violett II . . . . . . . . . $=18,4$,

Purpur . . . . . . . . . . $=21,6$.

Bei den farbigen Papieren gelten die angeg. relativen Helligkeitswerthe für diffuses Tageslicht. Die mit einem * bezeichneten Papiere sind aus der Papierfabrik von Schleicher und Schüll in Düren. Die beim Neutralgrau (Nr. 541) und beim hellen Silbergrau (Nr. 462) ang. Helligkeit gilt eigentlich für Proben, welche vor einigen Jahren bezogen wurden und sich durch einen reingrauen Ton auszeichneten. Die neuerdings von dieser Fabrik 
Ueber den Einfluss d. relativen Helligkeit u. d. Farbe d. Papieres etc. 567

gelieferten Papiere sind fast genau ebenso hell, doch hat das Neutralgrau (Nr. 541) einen gelblichen und Nr. 462 einen violettlichen Schimmer, der aber so gering ist, dass diese Papiere immerhin sehr brauchbar zu physiologisch-optischen Untersuchungen sind.

Die farbigen Papiere sind (mit Ausnahme des mit pikrins. Natron bestrichenen) echte Heidelberger Blumenpapiere.

Den Reduktionskurven Taf. VIII Fig. 1 sind die Werthe der Tabelle A zu Grunde gelegt. Mit Hülfe dieser Kurven können wir folgende Operationen ohne Rechnung ausführen:

(Hierbei wollen wir, zur Vereinfachung der Helligkeitsgleichung, von zwei Komponenten nur den Prozentsatz derjenigen Komponenten angeben, welcher direkt abgelesen wird, und die helleren Komponenten voranstellen ${ }^{1}$, es ist mithin $p \% . W / S=p \% W+(100-p) \% S$.)

I. Es wird die Normalhelligkeit $(x)$ einer Mischfarbe aus zwei (der Normalhelligkeit nach bekannten) Komponenten gesucht.

$$
\text { 'Z. B.: } 25,4 W / S=100 x \text {. }
$$

Den kontinuirlichen Uebergängen von $S$ nach $W$ entspricht die Kurve $S W$ Taf. VIII Fig. 1

für $y=25,4$ finden wir $x=25,0$.

[Die Rechnung ergiebt, nach Formel $1 \mathrm{~b}$, $x=\frac{25,4 \times 90,4+76,6 \times 2,6}{100}=24,9$ als Normalhelligkeit.]

Diese Methode dürfte für die Vergleichbarkeit der Resultate der Farbengleichungen von einigem Interesse sein ${ }^{2}$ ).

II. Es wird der Prozentsatz von zwei (bekannten) Komponentengesucht, der nöthig ist, um eine Mischfarbe von bestimmter Normalbelligkeit zu erzielen.

Es soll z. B. die Normalhelligkeit $10\left(\right.$ d. h. $\left.10 \% h_{N}\right)$ aus Pigmenten der Tabelle A hergestellt werden. - Wir müssen also das zu $x=10$ gehörige $y$ ablesen! Wie wir sehen, ist $x=10$ für $10,0 \mathfrak{W} / \subseteq: 8,3 W / S ; N / S 72,5$ u. s. w. Ebenso $x=50$ für $54,0 W / S ; 33,7 W / N ; 44,2 W / K ; G / S 12,6$ u. s. w.

1) Bei zwei farbigen Komponenten empfiehlt es sich, aus hier nicht näher zu erörternden Gründen, stets den Zusatz der brechbareren Farbe abzulesen, und diese nachzustellen. (Vergl. v. Graefe's Arch. XXX (1884), 2, pag. 36.)

2) Vergl. Centrbl. f. pr. Augh. (1885), Juli-Heft. 
III. Es soll eine bestimmte Mischung zweier Komponenten durch eine gleichhelle Mischung zweier anderer Komponenten ersetzt werden.

Z. B. Es soll ans Grün II und Weiss eine Mischfarbe hergestellt werden, die ebenso hell ist, wie eine gewisse Mischfarbe ans Karmin und Gelb $(G)$

$$
y \% W / G r_{\mathrm{II}}=K / G 57,5 \% \text {. }
$$

Wir suchen zuerst die Normalbelligkeit der Mischfarbe $K / G 57,5$ und finden $x=39,7$. Dem entspricht $17,4 W / G r_{\text {II }}$, mithin $y=17,4$ !

IV. Es soll die Normalhelligkeit $(h)$ eines Pigmentes gefunden werden ${ }^{1}$ ) aus der Gleichung:

wo $N, W, S$ bekannt sind.

$$
\left.p \% h / N=q^{\%} W / S^{1}\right)
$$

Im geg. Falle sei z. B. die Helligkeit des weissen Papieres Nr. 50 (von Schleicher und Schiull in Dïren) zu bestimmen.

Es wurde beobachtet $50,0 \mathrm{~h} / \mathrm{N}$ (Neutralgrau B.-K.) $=66,6 \mathrm{~W} / \mathrm{S}$ (d. h. weisser und schwarzer Bristolkarton). Der Mischung 66,6 W/S entspricht $x=61,0$. Um nun $h$ dureh Konstruktion zu finden, verbinden wir den Punkt der Abscisse $(y=0, x=N)$ mit dem Punkte, der die Koordinaten $y=50,0, x=61,0$ hat. Die Verlängerung schneidet die $(y=100$ entsprechende) Linie in einem Punkte, dessen Abscisse $x=92,6$ abgelesen wird. (In Fig. 1 Tab.VIII nicht ausgezogen.) $=92,65$.

Die Rechnung ergiebt $h=\frac{66,6 \times 90,4+33,4 \times 2,6-50.29,3}{50}$

(Der in Tabelle A mitgetheilte Werth $h=92,4$ ist das Mittel aus mebreren Einzelbestimmungen.)

Diese Beispiele dürften gentigen, um die Verwendbarkeit der Reduktionskurven zu zeigen.

1) Einfacher ist der Fall, wo entweder dasselbe Schwarz $(p \% h / S$ $\left.=q^{0} \% W / S\right)$ oder dasselbe Weiss $\left(p^{\circ} \% W / h=q^{\circ} \% W / S\right)$ benutzt wird. Für $S=0$ ist dann $h=\frac{q}{p} \cdot W\left[\right.$ resp. $\left.=\frac{q^{\prime}-p^{\prime}}{100-p^{\prime}} \cdot W\right]$.

Die auch von Aubert (dieses Arch. f. d. ges. Physiol. XXXI (1883), pag. 227-231) angewandte Methode der Herstellung eines identisch erscheinenden Grau (für ein zu bestimmendes graues Pigment), aus $h$ und $S$ (oder $W$ ) einerseits und $S$ und $W$ andererseits, giebt weit genauere Resultate als die direkte Einstellung $100 h=p \% W / S$, da hier ein etwaiger farbiger Schimmer des Pigmentes $h$ weit störender wirkt. D. Verf. 
Ueber den Einfluss d. relativen Helligkeit u. d. Farbe d. Papieres etc. 569

\section{Abhängigkeit der Sehschärfe von der relativen Helligkeit der Schriftzeichen und des betreffenden Grundes.}

Zur experimentellen Lösung dieser Frage schlug ich zwei Wege ein, indem ich als Hintergrund, auf welchem die Schriftzeichen sichtbar wurden, einerseits Tafeln von photometrisch bestimmter Helligkeit, andererseits rotirende Scheiben benutzte. Erstere Methode hat den grossen Vorzug der Bequemlichkeit, letztere gestattet aber die Helligkeit des Grundes nach Belieben zu variiren.

Messung der Sehschärfe an Tafeln.

Um durch verschiedene Oberflächenbeschaffenheit der benutzten Tafeln keine Fehlerquelle einzuftuhren, nahm ich reinweissen Bristolkarton $\left(h=90,4 \% h_{N}\right)$ und stellte durch Ueberpinselung mit Tusche (deren etwas bräunlicher Ton durch einen kleinen Zusatz von Indigo neutralisirt wurde) vier Schattirungen von reinem Grau her. Nach dem Trocknen wurde die Helligkeit der Flächen photometrisch (an rotirenden Scheiben) gemessen und nach Bedarf so lange regulirt, bis die Helligkeitsstufen die gewünschten Differenzen $\left(20 \% h_{N}\right)$ ergaben. Trotz aller Mühe gelang es mir nicht, die Helligkeiten der Tafeln völlig äquidistant zu machen; doch dürfte die Abweichung gering genug sein, um in diesem Falle vernachlässigt zu werden.

Die farbigen Tafeln wurden in ähnlicher Weise aus demselben weissen Bristolkarton hergestellt und ihre Helligkeit mit der der entsprechenden Heidelberger Blumenpapiere verglichen, welche bereits friiher von mir (s. o. Cit.) photometrisch bestimmt worden waren. Die vier benutzten Aquarellfarben waren: Karmin, Gummi-gutti, Scheel'sches Grün (arsenigs. Kupferoxyd) und Ultramarin.

Die Schriftzeichen wurden, genan entsprechend den Snellenschen Lesezeichen für Analphabeten $\mathbf{E}$ (fürr 6 Meter), ausgeschnitten und auf die Tafeln geklebt. Zu den schwarzen Schriftzeichen benutzte ich die oberste, geschwärzte Papierschicht des tiefschwarzen ${ }^{1}$ ) dicken Bristolkartons, zu weissen das weisseste

1) Die aufgeklebten Lesezeichen sahen viel akurater aus als die zur Probe gezeichneten, auch hat dieser schwarze Karton eine bedeutend ge- 
Visitenkartenpapier, zu grauen das erwähnte Neutralgrau (B.-K.), zu gränen das etwas bläulichgrüne Heidelb. Blumenpapier, zu purpurnen ein ausgeschnittenes Stiick meiner Farbentafel (D). Diese beiden Farben wählte ich, weil sie bei diffusem Tageslicht gleich intensiv und fast genau so hell sind, wie das benutate Neutralgrau.

Um den Einfluss der Refraktion za eliminiren, erhielten die Tafeln die Einrichtung, dass sie um ihren Mittelpunkt drehbar waren. Gemessen wurde die Sehschärfe für alle vier Hauptstellungen der Schriftzeichen (Oeffnung oben, unten, rechts, links) und das aritbmetische Mittel genommen.

Die drei Beobachter hatten einen normalen Farbensinn. An demselben Tage, an welchem die in der folgenden Tabelle B mitgetheilten Sehschärfemessungen angestellt wurden, lasen sie die Snellen'schen Schriftzeichen (s. o.) für 6 Meter:

$\mathrm{A}$ in 4,$5 ; \mathrm{B}$ in 7,$5 ; \mathrm{C}$ in 7,0 Metern.

(Um Lichtverluste zu vermeiden, wurde die Myopie des I. Beobachters $[M=3 D]$ nicht korrigirt.)

Die Messung der Helligkeit des diffusen Tageslichtes geschah am Anfang und am Ende jeder Beobachtungsreihe vermittelst eines von mir modifizirten Schattenphotometers nach Bertin-Sans (vergl. v. Graefe's Areh. f. Ophth. XXX (1884), 2 pag. 22 u. Fig. 4- , Taf. I), welches bei Anwendung von drei Schirmen (weiss, neutralgrau, dunkelgrau) gestattet, die Helligkeitsbestimmung auf ea. 3-4\% genau anzustellen. Als Einheit diente die Leuchtkraft einer Stearinkerze (zu vier auf's Pfund) in 1 Meter Abstand.

Die in nebenstehender Tabelle $A$ angegebenen Zahlen sind Mittelwerthe aus je drei vollständigen Beobachtungsreihen, also zwölf Einzelmessungen (s. o.).

Um dem möglichen Einwande zu begegnen, dass die Helligkeitsmessungen der granen und der farbigen Flächen (s. w. w.) vielleicht nicht genügend genau gewesen, oder dass die Helligkeit der bestrichenen Flächen nach der photometrischen Bestimmung sich geändert haben könne, versuchte ich eine analoge Messung der Sehschärfe mit schwebenden Lesezeichen und rotiren-

ringere Helligkeit $\left(2,6 \% h_{N}\right)$ als Tusche $\left(5,0 \% h_{N}\right)$, schwärzeste Tinte von A. W. Faber $\left(3,7 \% h_{N}\right)$ und Druckerschwärze $(3,7 \% h N)$, was in diesem Falle von Wichtigkeit war. 
Ueber den Einfluss d. relativen Helligkeit u. d. Farbe d. Papieres etc. 571

Tab. B. Sehschärfe bei differenter Helligkeit der Tafeln Beleuchtung: Diffuses Tageslicht (entspr. ca. 50 Meterkerzen).

\begin{tabular}{|c|c|c|c|c|c|c|c|}
\hline \multicolumn{2}{|c|}{$\left.\begin{array}{c}\text { Helligkeit } \\
\text { des Grundes }\end{array}\right\} G=$} & 96,3 & 90,4 & 61,8 & 41,4 & 26,4 & 6,8 \\
\hline $\begin{array}{c}\text { Lesezeichen } \\
\qquad Z\end{array}$ & Beob. & 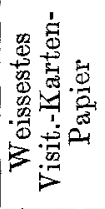 & 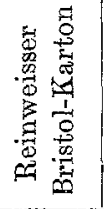 & $\stackrel{\mathscr{Z}}{\vec{E}}$ & 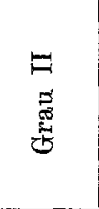 & 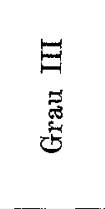 & 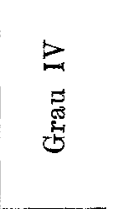 \\
\hline \multirow[t]{3}{*}{$\begin{array}{l}\text { Schwarz } \\
2,6 \% h_{N}\end{array}$} & $\begin{array}{l}\mathrm{A} \\
\mathrm{B} \\
\mathrm{C}\end{array}$ & $\begin{array}{l}5,0 \\
9,0 \\
8,0\end{array}$ & $\begin{array}{l}4,5 \\
8,0 \\
6,0\end{array}$ & $\begin{array}{l}3,7 \\
6,5 \\
5,3\end{array}$ & $\begin{array}{l}3,4 \\
6,0 \\
5,0\end{array}$ & $\begin{array}{l}2,7 \\
5,5 \\
4,5\end{array}$ & $\begin{array}{l}2,2 \\
3,0 \\
3,2\end{array}$ \\
\hline & Mittel & 7,2 & 6,2 & 5,0 & 4,8 & 4,2 & 2,8 \\
\hline & $(G-Z)$ & $(93,7)$ & $(87,3)$ & $(59,2)$ & $(38,8)$ & $(23,8)$ & $(4,2)$ \\
\hline \multirow[t]{3}{*}{$\begin{array}{c}\text { Neutralgrau } \\
29,1\end{array}$} & $\begin{array}{l}\mathrm{A} \\
\mathrm{B} \\
\mathrm{C}\end{array}$ & $\begin{array}{l}4,0 \\
6,5 \\
6,5\end{array}$ & $\begin{array}{l}3,8 \\
6,3 \\
5,3\end{array}$ & $\begin{array}{l}3,6 \\
6,2 \\
5,2\end{array}$ & $\begin{array}{l}2,5 \\
6,0 \\
4,3\end{array}$ & $\begin{array}{l}1,5 \\
2,3 \\
2,8\end{array}$ & $\begin{array}{l}3,0 \\
6,5 \\
4,8\end{array}$ \\
\hline & Mittel & 5,7 & 5,1 & 5,0 & 4,3 & 2,2 & 4,8 \\
\hline & $(G-Z)$ & $(67,2)$ & $(61,2)$ & $(32,7)$ & $(12,3)$ & $(-2,7)$ & $(-22,3)$ \\
\hline \multirow{3}{*}{$\begin{array}{c}\text { Purpur } \\
28,5\end{array}$} & $\begin{array}{l}\mathrm{A} \\
\mathrm{B} \\
\mathrm{C} \\
\end{array}$ & $\bar{z}$ & $\begin{array}{l}3,3 \\
7,2 \\
7,0\end{array}$ & $\begin{array}{l}3,3 \\
7,0 \\
5,5\end{array}$ & $\begin{array}{l}2,0 \\
5,3 \\
3,4\end{array}$ & $\begin{array}{l}2,3 \\
4,5 \\
4,2\end{array}$ & $\begin{array}{l}3,8 \\
5,5 \\
6,0\end{array}$ \\
\hline & Mittel & - & 5,8 & 5,3 & 3,6 & 3,6 & 5,1 \\
\hline & $(G-Z)$ & - & $(61,9)$ & $(35,3)$ & $(12,9)$ & $(-1,9)$ & $(-21,7)$ \\
\hline \multirow[t]{3}{*}{$\underset{29,3}{\text { Grün II }}$} & $\begin{array}{l}\mathrm{A} \\
\mathrm{B} \\
\mathrm{C}\end{array}$ & E & $\begin{array}{l}2,3 \\
6,2 \\
4,3\end{array}$ & $\begin{array}{l}2,8 \\
5,7 \\
4,2 \\
\end{array}$ & $\begin{array}{l}2,0 \\
2,8 \\
3,5\end{array}$ & $\begin{array}{l}2,0 \\
5,8 ! \\
3,5\end{array}$ & $\begin{array}{l}2,8 \\
6,8 \\
6,1\end{array}$ \\
\hline & Mittel & - & 4,3 & 4,2 & 2,8 & 3,8 & 5,2 \\
\hline & $(G-Z)$ & - & $(61,0)$ & $(32,5)$ & $(12,1)$ & $(-2,9)$ & $(-22,5)$ \\
\hline
\end{tabular}

den Scheiben als Hintergrund. Da ich eine grössere Reihe von Pigmenten (darunter anch Heidelberger Blumenpapiere) wiederholt nach mehreren Methoden auf ihre Helligkeit untersucht und eine fuir unseren Zweck genügende Uebereinstimmung der Resultate erhalten hatte, so war es leicht, mit Hülfe der oben entwickelten Formel 1b, oder bequemer vermittelst der Reduktionskurven Fig. 1 Taf. VIII, jede erforderliche Normalhelligkeit dem grauen, resp. farbigen Hintergrunde zu geben. 
Zu diesem Zwecke spannte ich je zwei sehr fe ine graue Seidenfäden kreuzweise über einen schmalen Ring aus starkem Karton (s. Fig. 2 Taf. IX) und klebte darauf je vier Lesezeichen (identisch den Snellen'schen für 6 Meter, wie oben). Diese Lesezeichen wurden aus sehr diunnem, steifen Karton geschnitten, nachdem dieser mit der obersten geschwärzten Schicht des tiefschwarzen Bristolkartons (resp. mit Heidelberger Blumenpapieren) überzogen worden war. Der feine beim Ausschneiden entstandene weisse Rand wurde vorsichtig mit der Farbe der betr. Buchstaben bepinselt, so dass auch bei einer seitlichen Stellung des Beobachters keine Spur des Randes sichtbar war. Ein Stativ, dessen Arm in jeder Richtung festgestellt werden konnte, und der an seinem Ende eine kleine Federklemme trug, stand seitlich vor dem Rotationsapparat. Vermittelst der Federklemme konnten die Ringe mit den Lesezeichen leicht passend eingestellt und rasch gewechselt werden. Bei der Messung der Sehschärfe erhielt ich anfangs widersprechende Resultate, bis ich folgende Vorsichtsmassregeln streng befolgte: 1 . Die als Hintergrund dienende Scheibe muss gleichmässig beleuchtet sein und darf nicht vom Ringe beschattet werden. 2. Die Ebene der Lesezeichen und die Scheibe diurfen keine Reflexe in der Richtung der Visirlinie zeigen. 3. Die Richtung der Visirlinie muss mit der des Helligkeitsminimums (für die geg. Stellung zum auffallenden Lichte) zusammenfallen (s. o.).

Bei sorgsamer Ausführung bietet diese Methode den Vortheil, dass dieselben Lesezeichen benutzt werden, mithin kleine Grössenabweichungen, welche bei Tafeln leicht vorkommen, keine Fehlerquelle bilden. [Bei künstlicher Beleuchtung müssen zwei Lichtquellen passend angebracht werden, um die Scheibe gleichmässig zu beleuchten, dennoch giebt dieses Verfahren selbst dann schwankende Resultate, da es kaum möglich ist, vermittelst der Lampen die Scheibe und die Lesezeichen gleich intensiv zu beleuchten]. Am günstigsten (wie bei allen Prüfungen der Sehschärfe oder des Farbensinnes) ist Oberlicht. 
Ueber den Einfluss d. relativen Helligkeit u. d. Farbe d. Papieres etc. 573

Tab. C. Sehschärfemessungen mit rotirenden Scheiben als Hintergrund.

(Diffuses Tageslicht $=58-63$ Meterkerzen.)

1. Grauer Hintergrund.

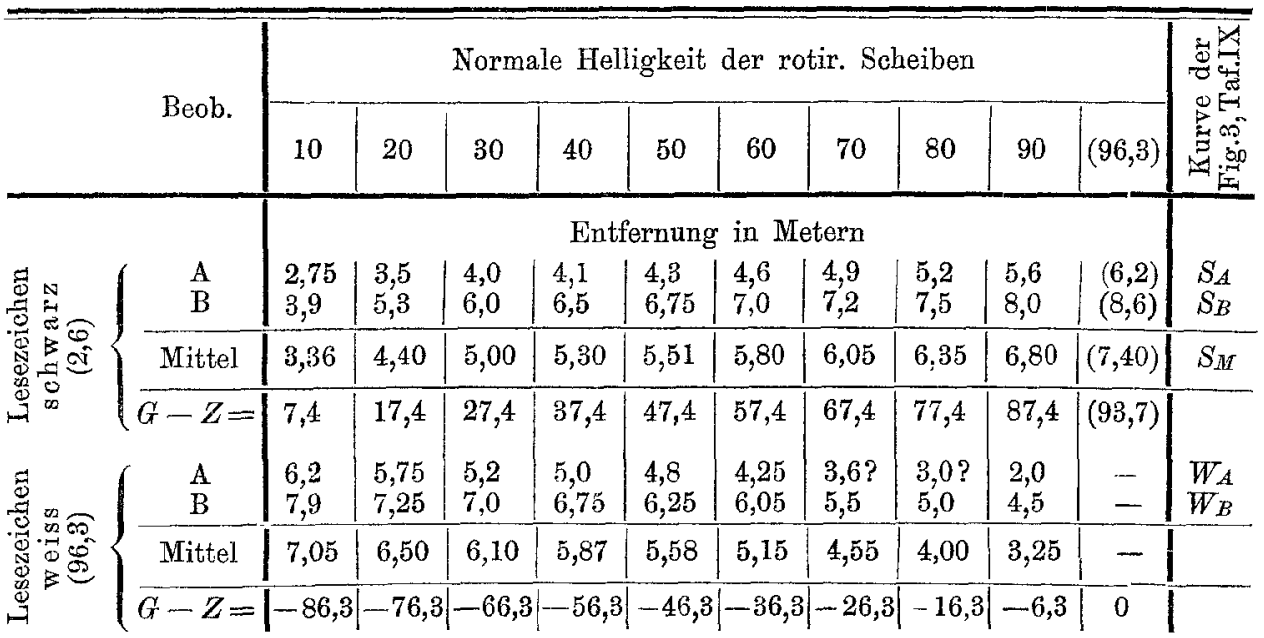

2. Farbige Scheiben als Hintergrund.

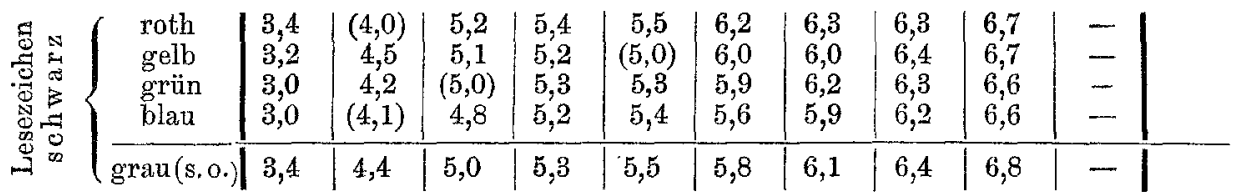

Anm. Die hier angeg. Zahlen sind reduzirte Mittelwerthe beider Beob. (s. w. u.). (Die eingeklammerten Werthe entsprechen der Sehschärfe bei maximalem Prozentsatz der Farbe des Grundes.)

3. Sehschärfemessungen an grauen Scheiben bei verschiedener Beleuchtungsstärke.

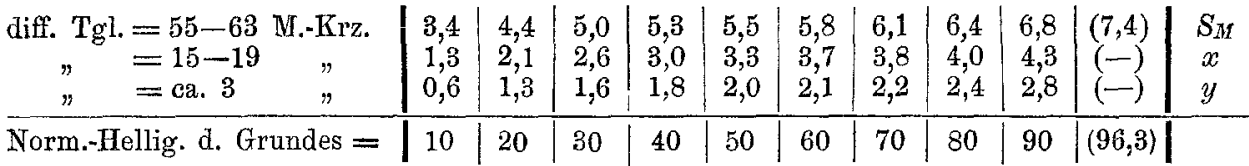

In Bezug auf die angeführten Sehschärfe-Tabellen ist noch Folgendes zu bemerken:

Tab. B. Die gleichfalls beobachteten Werthe für weisse Lesezeichen sind fortgelassen, weil die Resultate wegen mangelhafter Herstellung dieser Zeichen unsicher waren. Es war mir 
nämlich nicht möglich, gute weisse Zeichen zu erhalten, indem selbst der feine Visitenkarten-Karton sich nicht befestigen liess, ohne entweder über die Fläche der Tafel herauszuragen und dann störende Handschatten zu geben, oder beim Einlassen (resp. Einpressen) eine Grenzlinie zu geben, welche bei geringen Helligkeitsdifferenzen zwischen Zeichen und Grund sehr störend wirkten. Feines weisses Papier hatte zwar diesen Uebelstand nicht, liess aber den dunklen Grund durchschimmern, wodurch die Helligkeit der Lesezeichen in schwer zu kontrollirender Weise verändert wurde. Die aus dem feinen weissen Bristol-Visitenkartenkarton geschnittenen Lesezeichen, welche auf die ausgespannten Fäden (Fig. 2, Taf. IX) geklebt wurden, sind frei von diesen Mängeln.

Tab. C, 2. Der besseren Uebersicht wegen wurden die beobachteten Werthe an farbigen rotirenden Scheiben auf die an grauen Scheiben Tab. C, 1 reduzirt. Es war nämlich, da die Versuche nicht bis zur Ermiidung der Angen fortgesetzt werden durften, nothwendig, die einzelnen Beobachtungsreihen an verschiedenen Tagen anzustellen. Es wurde daher stets die Sehschärfe für einen farbigen und einen gleichhellen grauen Grund gleichzeitig gemessen. Zu diesem Zwecke befestigte ich an der rotirenden Achse eine grössere schwarz-weisse und darüber eine kleinere farbige (z. B. schwarz-rothe) Scheibe ${ }^{1}$ ). Der Ring mit den Lesezeichen stand nun vor der einen Hälfte der rotirenden Scheibe (bei der ersten Messung vor der linken, bei der zweiten vor der rechten), so dass je zwei Lesezeichen auf farbigem und je zwei anf granem Grunde sichtbar waren.

Die Reduktion auf die Werthe der Tab. C, 1 wurde in folgender Weise vorgenommen:

Es wurde z. B. beobachtet für eine Helligkeit des Grundes $h=10 \% h_{N}$.

A.

B.

Farbe des Hintergrundes: roth grau roth grau

beobachtet $D^{\prime}=$. . . $\quad 1,9 \quad 2,0 \quad 3,3 \quad 3,1$

(Tab. C, 1) $D=$. . . $\quad$ - $\quad 2,75 \quad$ - 3,9

1) Die Sektoren wurden vermittelst der Reduktionskurven Fig. 1, Taf.VIII so eingestellt, dass dieselbe verlangte Normalhelligkeit erhalten wurde. 
Ueber den Einfluss d. relativen Helligkeit u. d. Farbe d. Papieres etc. 575

Hieraus ergiebt sich für A . . . $D=\frac{1,9.2,75}{2,0}=26,611$ für B . . $\quad$. $D=\frac{3,3.3,9}{3,1}=4,133$

Im Mittel $D=3,4$ (statt 3,37 gesetzt).

Aus den Untersuchungsresultaten ergiebt sich nun Folgendes:

I. Die Sehschärfe wächst mit der Helligkeitsdifferenz zwischen den Schriftzeichen und dem Grunde, doch ist die Zunahme nicht gleichmässig.

Anfangs (bei dunkelm Grunde und schwarzen Lettern) wächst die Sehschärfe rasch, dann immer langsamer, um schliesslich wieder stärker anzusteigen. Bis zum Flexionspunkt, der etwa $(G-Z)=54 \% h_{N}$ entspricht, erinnert die Sehschärfenkurve (Fig. 3, Taf. IX) an eine Hyperbel, die um so flacher wird, je mehr die Beleuchtungsstärke abnimmt. Auffallend ist das steilere Ansteigen der Kurven fiur hellen Hintergrund $(S=75$ bis 94$)$; und zwar ist die Zunahme der Sehschärfe beim weissesten Papiere $\left(h=96,3 \% h_{N}\right)$ gegenüber dem etwas dunkleren reinweissen Karton $(90,4)$ um so deutlicher, je schwächer die Beleuchtung war!

Hierbei scheinen jedoch kleine individuelle Verschiedenheiten vorzukommen, denn bei Kontrolversuchen an 13 Personen $(S>1)$ fanden zwei Beobachter kaum einen Unterschied in der Erkennbarkeit schwarzer Lettern $^{1}$ ) auf weissem Bristolkarton $(h=90,4)$ und weissestem Visitenkartenpapier $(h=96,3)$, doch trat die Differenz der Sehschärfe ausnahmslos stärker hervor, wenn kleinere Lesezeichen angewandt wurden, besonders bei schwächerer Beleuchtung. Dieser Umstand scheint mir von praktischer Bedeutung.

Um die von Brücke ${ }^{2}$ ) benutzte Methode der Helligkeitsbestimmung von farbigen Papieren zu versuchen, hatte ich mir

1) Bei dunkelgrauen Lettern (Tusche, sehlechte Druckerschwärze etc.) trat die Differenz bei allen Beobachtern deutlicher hervor, als bei schwarzen Lettern.

2) Brü cke: Wiener Akad. Ber. Bd. 84, Abth. III, pag. 425, - Refer. in Michel's Jahresb. f. Ophth. 1881, pag. 128. 
mit vieler Mühe eine (photometrisch kontrolirte) HelligkeitsTafel mit 23 fast aequidistanten Helligkeitsabstufungen (von Sehwarz nach Weiss) hergestellt. Zu meiner Ueberraschung fand ich, dass die Uebergänge, welche in $1 / 2$ bis 1 Meter Entfernung gleichmässig erschienen, bei 2 Metern und weiter, ganz ungleich aussahen. Von ca. $h=35 \% h_{N}$ an schien die Tafel aus einer hellen und einer dunkeln Hälfte zu bestehen. Von der mittleren Grenze an nabmen jetzt die Helligkeitsdifferenzen rasch ab, so dass bei den hellsten wie bei den dunkelsten Schattirungen keine oder nur minime Abstufungen sichtbar waren. Zur Kontrole wandte ich an meinem Farbenmesser ${ }^{1}$ ) Cylindermäntel mit graduellen und mit kontinuirlichen Uebergängen von Schwarz nach Weiss an und erhielt auch hier, wenn auch weniger frappant, dasselbe Resultat. Bei herabgesetzter Beleuchtung tritt diese Erscheinung in einer relativ kleineren Entfernung ein.

Diese Beobachtungen stehen im Einklang mit den erwähnten Resultaten der Sehschärfemessungen. Ein Zusammenhang beider Erscheinungen ist sehr wahrscheinlich.

II. Die Farbe des Grundes seheint (bei sehwarzen ${ }^{2}$ ) Lettern) olıne wesentlichen Einfluss auf die Sehschärfe $\mathrm{zu}$ sein, indem (wenigstens bei meinen Messungen) nur die Helligkeitsdifferenz zwischen den Lesezeichen und dem Grunde den Unterschied bedingt. Dieses tritt aber erst dann deutlich hervor, wenn man Flächen von photometrisch bestimmter gleicher Helligkeit anwendet. Aus den zum bequemeren Vergleich reduzirten Werthen der Tab. C, 2 ist keine Farbe als besonders günstig zu erkennen.

Bei künstlicher Beleuchtung gestaltet sich die Sache wesentlich anders. Von einer Reihe von farbigen Papieren (resp. Mischfarben an rotirenden Scheiben), welche bei diffusem Tageslicht gleich hell sind, werden natürlich die Farben, deren prävalirende

1) Beschrieben u. a. in v. Graefe's Arch. f. Opht. 1884, II, pag. 35 (Fig. 1A und 3, Taf. I).

$\mathrm{Zu}$ obigen Versuchen wurden Cylindermäntel von $20 \mathrm{~cm}$ Höhe benutzt.

2) Graue Lettern, etwa Neutralgrau No. 542 (s. o.), konnte ich hierbei nicht anwenden, da die verschiedene Oberflächenbeschaffenheit (des gekörnten grauen Papieres und der summetartig erscheinenden rotirenden Scheiben) bei Personen mit guter Sehschärfe zu störend wirkte, besonders bei kleinen Helligkeitsdifferenzen $(G-Z)$. 
Ueber den Einfluss d. relativen Helligkeit a. d. Farbe d. Papieres etc. 577

homogene Komponenten der Beleuchtungsfarbe isoch rom sind, relativ heller und ihre Gegenfarben relativ dunkler aussehen. Da also für schwarze Lettern bei künstlicher Peleuchtung die Helligkeit des farbigen Grundes (je nach der betreffenden Farbe des Grundes und der Lichtquelle) in versehiedener Weise verändert wird, so wird auch die Sehschärfe entsprechend grösser oder kleiner sein müssen; ich habe aber nicht bemerken können, dass schwarze Lesezeichen auf farbigem Grunde deutlicher wahrgenommen werden als auf gleichbellem grauen Grunde. Für diffuses Tageslicht geht dieses auch aus Tab. C, 2 hervor, indem die dort vorhandenen Differenzen der Sehweite noch innerhalb der Grenzen der Beobachtungsfehler liegen. - Es könnte auf den ersten Blick scheinen, als müsse gelbliches Papier bei Kerzen- oder Lampenlicht bedentend heller sein als hellgraues Papier, das bei diffusem Tageslicht gleich hell war; das ist jedoch nicht der Fall. Man mache nur folgende Probe: Ein entsprechend eingerichtetes Spektroskop richte man auf eine rotirende schwarz-weisse Scheibe, welche von einer kleineren gelblichen Scheibe zum Theil bedeckt wird, so dass die Spektren gleichzeitig sichtbar sind. Regulirt man nun die Sektorenbreite der schwarz-weissen Scheibe so, dass die praevalirenden Komponenten in beiden Spektren (hier gelb und orange) gleich intensiv erscheinen (bei diffusem Tageslicht), so wird bei Kerzen- oder Lampenlicht kaum oder nur eine minime Verstärkung dieser Komponenten beim gelblichen Papier gegenüber denen beim entsprechenden Grau bemerkbar sein, während jetzt die brechbareren Komponenten relativ schwächer sind, so dass die Gesammthelligkeit der gelblichen Fläche nicht merklich grösser ist als die der grauen.

Die Sehschärfemessungen bei künstlicher Beleuchtung ergaben so schwankende Resultate, dass ich auf die Wiedergabe der Resultate verzichte. Die Schuld muss ich der erwähnten Schwierigkeit beimessen: die rotirenden Flächen und die Lesezeichen gleichmässig zu beleuchten. Die von mir zuerst benutzten farbigen Tafeln (s. o.) habe ich später nicht verwendet, da dort die bei der Herstellung der Lesezeichen etwa begangenen Febler (in der Grösse der Lettern) bei den minimen Unterschieden, auf die es hier ankam, leicht hätten zu falschen Resultaten Anlass geben können. 
III. Von besonderem Interesse ersehien mir die Frage: bei welcher Farbe des Grundes ermüdet das Auge beim Lesen und Schreiben am wenigsten?

Die Versuche stellte ich in der Weise her, dass ich an Snellen's Tafeln meine Sehschärfe bestimmte, nachdem ich bereits [eine Stunde etwa] gelesen und geschrieben hatte ${ }^{1}$ ) (auf weissem Grunde). Darauf schrieb, zeichnete und las ich eine balbe resp. eine ganze Stunde auf farbigem Grunde ${ }^{2}$ ) und mass wieder die Sehschärfe. An jedem Tage wurde nur eine solche Beobachtung angestellt. Je nachdem ich ïberhaupt frischer oder abgespannter war, fielen die Resultate mehr oder weniger schwankend aus, doch zeigte sich deutlich Folgendes:

Farbiger Grund ermüdete das Auge mehrals weisser, und zwar um so mehr, je intensiver die farbigen Nachbilder sind, welche die betreffende Farbe erregt. So ermüdete mein Auge mehr bei rosa und bei grünem Grunde als bei gelbem und blanem, am wenigsten bei weissem.

Fasse ich das praktische Ergebniss meiner Beobachtungen zusammen, so muss ich mich gegen den von Javal ${ }^{3}$ ) gemachten und auch vom hygienischen Kongress in Turin (1880) angenommenen Vorschlag: dass Schulbuicher auf gelblichem (oder gar gelbem) Papier gedruckt werden sollten, aussprechen; dagegen stimme ich völlig mit $\mathrm{C}_{0} \mathrm{~h}^{4}$ ) darin überein, dass Schulbücher auf recht weissem (nicht durchscheinenden) Papier mit genügend grossen Lettern gedruckt werden mitsssen. Eine Helligkeits differenz von $80 \% h_{N} z w i s c h e n$ Lettern und Grund (also wie bei Snellen's Leseproben etwa) sollte das zu fordernde Minimum sein.

Da, wie bereits oben bemerkt wurde, die ungleiche Beleuch-

1) Das geschah, um die Adaption der Augen zu einer möglichst constanten zu machen.

2) Benutzt wurden farbige Papiere von der Intens. der gebräuchlichen Löschblätter und mattere.

3) Javal: De la couleur du papier d'impression au point de vue hygiènique. Société de biologie. 21. Febr. 1879. Vergl. Revue d'hygiène 1879, pag. 410.

4) Cohn: Die Hygiene des Auges in den Schulen. Wien 1883, Urban und Schwarzenberg, pag. 160. 
Ueber den Einfluss d. relativen Helligkeit u. d. Farbe d. Papieres etc. 579

tung der Scheiben resp. Tafeln eine störende Fehlerquelle bildet, in der angenärztlichen Praxis die ktinstliche Beleuchtung der Leseproben nicht zu umgehen ist, so sollten die betr. Tafeln von zwei, seitlich befindlichen Lampen beleuchtet werden, welche mit passend angebrachten Reflectoren versehen sind. Ganz vorzüg lich sind hiezu Hohlspiegel (von sphärischer oder parabolischer Form), welehe aus grünlichblauem Glase bestehen und an der convexen Seite versilbert sind. Das von solchen Reflectoren zurückgeworfene Lampenlicht ist zwar etwas weniger hell als das von Metallreflectoren, dagegen kann man durch passende Dicke des Glases und entsprechende Grösse der Reflectoren ein sehr schön weisses Licht erhalten, bei welchem man sogar Prüfungen des Farbensinnes anstellen kann. Aehnlich wirkt eine hohle Glaskugel, welche man mit destillirtem Wasser füllt, dem etwas Indigo ${ }^{1}$ ) zugesetzt ist. Hier hat man es durch Concentrirung resp. Verdïnnung der farbigen Lösung ganz in der Hand, den Farbenton des durchgelassenen Lampenlichtes zu verändern. Um zu entscheiden, ob das Licht nahezu reinweiss ist, braucht man nur am Farbenkreisel zwei Paar complementärer Papiere (z. B. roth-blaugrün und gelb-blau) so einzustellen, dass sie bei diffusem Tageslicht eine graue Mischung geben. Ist dieses bei der zu kontrolirenden künstlichen Beleuchtung für dieselbe Sectorenbreite der Fall, so ist das betr. künstliche Licht genügend nentralisirt $d$. h. farblos.

Mit wachsender Beleuchtungsstärke nimmt, wie wir gesehen baben, die Differenz der Schärfe für etwas versehieden helle weisse Papiere verhältnissmässig ab, daher ist es von Wichtigkeit, dass die im Zimmer freihängenden Leseproben, deren Papier mit der Zeit gelblich, also dunkler za werden pflegt, ge nügend hell beleuchtet werden. Bei genaueren Messungen sollte der Einfluss des jetzt dunkleren Grundes in Rechnung gezogen werden. - $\mathrm{Cohn}^{2}$ ) hat experimentell gefunden, dass

1) Bräcke empfiehlt (zur Farblosmachung des Lampenlichtes) als Füllung der Glaskugel eine schwache Lösung von Kupfervitriol. (Die Farbenlehre im Hinblick auf Kunst und Kunstgewerbe. Braunschweig 1874, pag. 53.)

2) Cohn: Ueber den Beleuchtungswerth der Lampenglocken. Wies baden 1885 . Bergmann, pag. 71 . 
erst bei 50 Meterkerzen das Auge etwa so gut und so bequem ohne Accommodations-Anstrengung sehenkann, wie bei Tage. Als Minimum der Helligkeit beim Arbeiter fordert Cohn 10 Meterkerzen, was gewiss nicht za viel ist. [Nach Prof. Weber (eit. von Cohn) hat ein horizontal liegendes Stuck Papier, das von einer Kerzenflamme, die $15 \mathrm{~cm}$ höher und seitwärts steht, beleuchtet wird, dieselbe Helligkeit wie bei 10 Meterkerzen.]

In Bezug auf weisse Lettern auf dunklem Grunde bin ich zu keinem positiven Resultate gekommen (ich habe diese Frage als nebensächlich, auch nur flüchtig behandelt). Wie der Verlauf der Curven $W_{A}$ und $W_{B}$ im Vergleich mit $S_{A}$ und $S_{B}$ (letztere entsprechen dunklem Grunde) zeigt, scheint bei kleinen Helligkeitsdifferenzen Weiss auf Schwarz besser, bei grösseren Differenzen schlechter erkannt zu werden, als Schwarz auf Weiss ${ }^{1}$ ). Jedenfalls ist die Bestimmung der Entfernung, in welcher schwarze Lettern (auf weissem Grunde) erkannt werden, weniger Schwankungen angesetzt, als die bei weissen Lettern!

Vergleichen wir noch die Messungen mit neutralgrauen und gleichhellen purpurnen und grünen Lezezeichen (Tab. B), so sehen wir, dass bei geringen Helligkeitsdifferenzen zwischen Lettern und Grund farbige Lesezeichen bedentend weiter erkannt werden, was selbstverständlich ist. Bei grösseren Helligkeitsdifferenzen ist der Unterschied geringer; hier wirkt also die Farbe als solche nicht mehr so, als die Helligkeit.

Es würde dies dafür sprechen, dass man bei der quantitativen Prüfung des Farbensinnes nach der Donders'schen Methode nicht einen schwarzen, sondern einen reing rauen, gleich hellen Grund zu wählen hätte. Da letztere Bedingung nur für eine constante Beleuchtungsart durchführbar ist, wenn man fertige Pigmentflächen benutzen will, so dïrften die schwebenden farbigen Lesezeichen (Fig. 2, Taf. IX) mit rotirenden Scheiben als Hintergrund in gewissen Fällen brauchbar sein, wo man die Zeit hat, die Sectoren entsprechend einzu-

1) Cohn fand, dass schwarze Buchstaben weiter erkannt würden. Cit. (s. w. o.) pag. 141. 
Ueber den Einfluss d. relativen Helligkeit u. d. Farbe d. Papieres etc. 581

stellen. Natïrlich müsste die Helligkeit der betr. Pigmentfarben für die benutzte Beleuchtung bestimmt sein.

Leider lassen sich meine Beobachtungsresultate nicht mit den interessanten Untersuchungen von Aubert, $\mathrm{Posch}, \mathrm{Cohn}$, Kramer, Charpenter u. A. iiber die Abhängigkeit der Sehschärfe von der Beleuchtungsstärke vergleichen, da die benutzten Methoden und das verfolgte Ziel zu verschieden sind.

Vorstehende Untersuchungen sollten auch nur eine kleine Lücke ausfüllen helfen, auf welche $\mathrm{Cohn}$ in seiner bekannten Arbeit (Ueber die Hygiene des Auges in den Schulen, pag. 160) hingewiesen hat.

\section{Erklärung der Abbildnngen auf Tafel VIII n. IX.}

Fig. 1. Reductionscurven zur Bestimmung der Normalhelligkeit von Mischfarben.

Die Abscisse $x$ bedeutet die Normalhelligkeit.

Die Ordinate $y$ bedeutet den beobachteten Zusatz von Weiss $(+y)$ oder Schwarz $(-y)$. Vergl. Taf. A u. d. folg. Text.

Fig. 2. Ring mit Lesezeichen (für rotir. Scheiben als Hintergrund). In 1/2 nat. Grösse. [Die vier Lesezeichen waren bei einigen Ringen unter sich gleich, bei anderen verschieden (z. B. schwarz, grau, grün, purpur). In letzterem Falle wurde die Sehschärfe für 4 Stellungen des Ringes $A, B, C, D$ oben) bestimmt und das Mittel genommen].

Fig. 3. Sehschärfenkurven (vergl. Tab. B u. C).

Die Abscisse ist die Helligkeitsdifferenz zw. Lesezeichen $u$. Grund.

Die Ordinate giebt die Entfernung an, in welcher die Lettern erkannt wurden (entspr. Snellen 6 Meter).

I. Schwarze Lettern auf Tafeln von verschiedener Helligkeit. Die ïbrigen Kurven entsprechen rotirenden Scheiben als Hintergrund. $W_{A}$ u. $W_{B}$ weisse Lettern.

$S_{A}, S_{B}, S_{M}$ (Mittel). Schwarze Lettern. Beleuchtung entsprechend 58-63 Meterkerzen.

$x$ schwarze Lettern. Beleuchtung etwa 15-19 Meterkerzen.

$y \quad, \quad$, $"$ ca. 3 " 


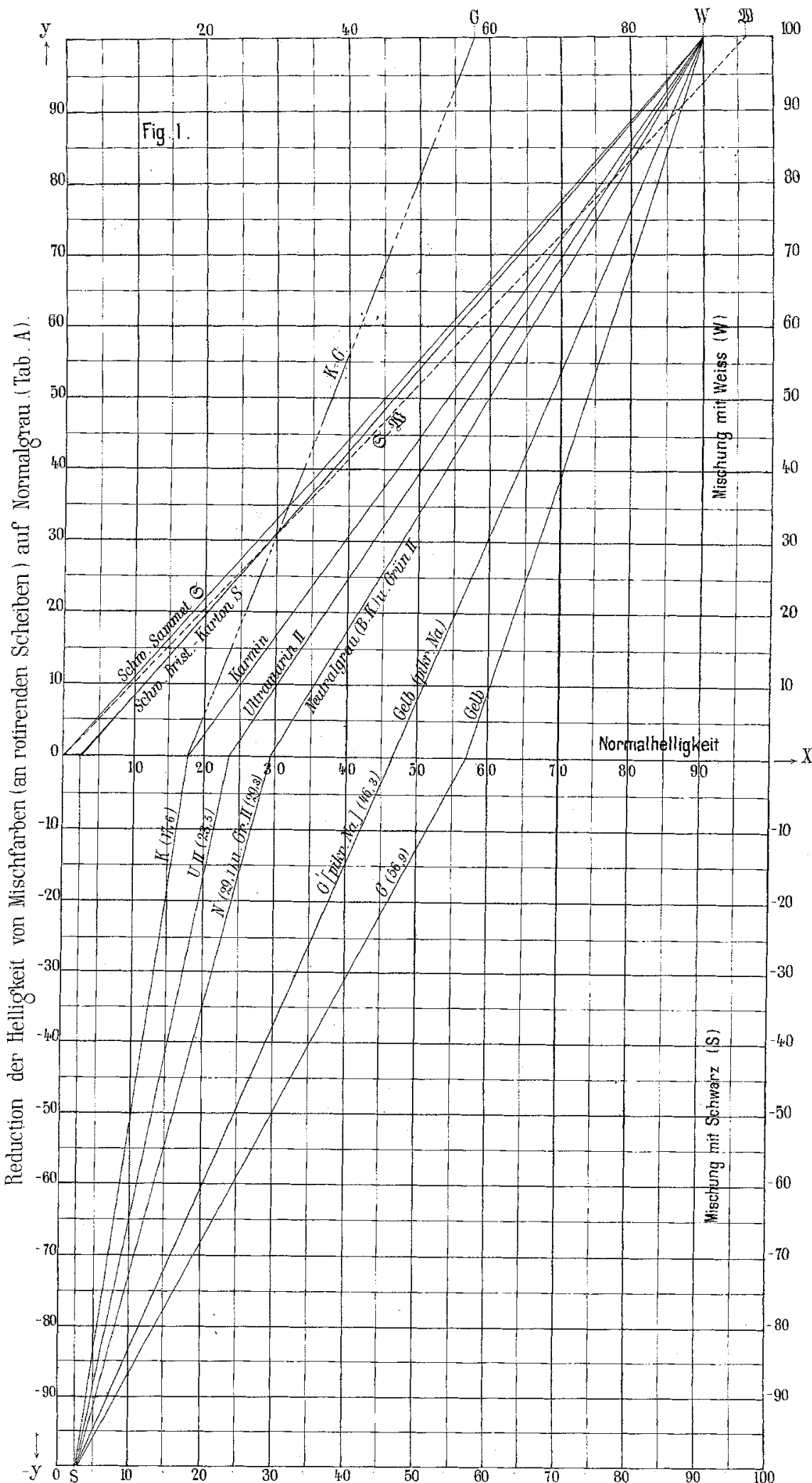


Fig. 2.

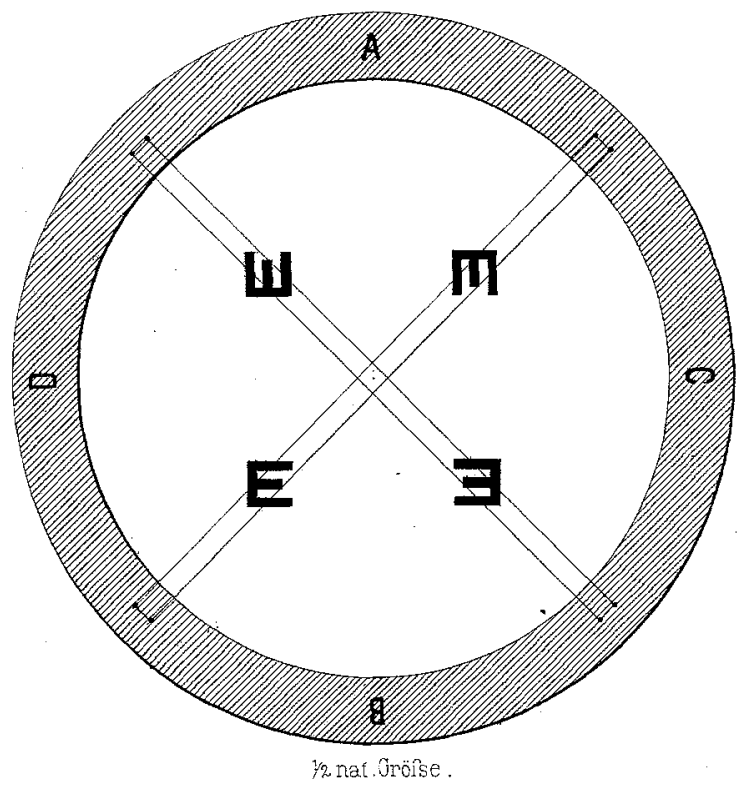

Fig. 3.

Sehschärfe bei versch. Hellight. des Grundes.

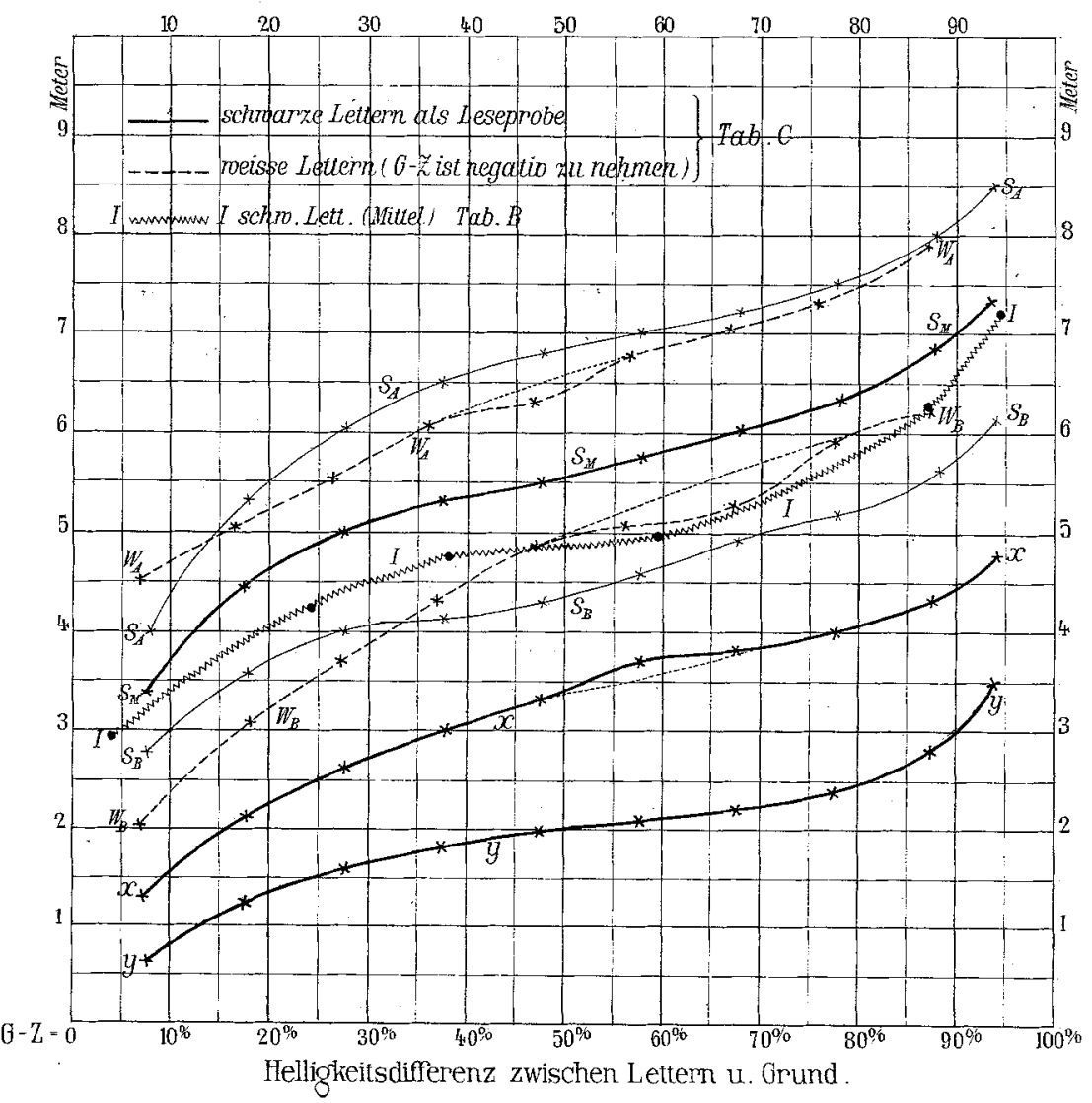

